\title{
Progress in Cavity QED with Single Trapped Atoms
}

\author{
Russel. L. Miller, H. J. Kimble, Boca Andreea, Kevin M. Birnbaum, Allen D. Boozer, \\ Tracy E. Northup \\ Department of Physics, California Institute of Technology, USA
}

\begin{abstract}
We report on recent progress in our lab involving cavity quantum electrodynamics with optically trapped atoms $[1,2,3,4]$.

In particular, we will focus on a recent measurement [1] of the Vacuum-Rabi splitting for one atom strongly coupled to the field of a high finesse optical resonator. This splitting is characteristic of the normal modes in the eigenvalue spectrum of the atom-cavity system. A new Raman scheme for cooling atomic motion along the cavity axis enables a complete spectrum to be recorded for an individual atom trapped within the cavity mode, in contrast to all previous measurements in cavity QED that have required averaging over many atoms.
\end{abstract}

We will also report on observation of photon blockade in the light transmitted by the atomcavity system. Excitation of this system by a first photon "blocks" the absorption of a second photon, thereby converting an incident Poissonian stream of photons into a subPoissonian, antibunched stream, as confirmed by measurements of photon statistics of the transmitted field. These observations also serve as a means for characterizing the motion of the trapped atom.

[1] A. Boca, R. Miller, K. M. Birnbaum, A. D. Boozer, J. McKeever, and H. J. Kimble, Phys. Rev. Lett. 93, 233603 (2004).

[2] J. McKeever, A. Boca, A. D. Boozer, J. R. Buck, and H. J. Kimble, Nature 425, 268 (2003).

[3] J. McKeever, A. Boca, A. D. Boozer, R. Miller, J. R. Buck, A. Kuzmich, and H. J. Kimble, Science 303, 1992 (2004).

[4] J. McKeever, J. R. Buck, A. D. Boozer, and H. J. Kimble, Phys. Rev. Lett. 93, 143601 (2004). 